\title{
Transfer of multidrug-resistant bacteria to healthcare workers' gloves and gowns after patient contact increases with environmental contamination
}

\author{
Daniel J. Morgan, MD, Elizabeth Rogawski, BS, Kerri A. Thom, MD, MS, J. Kristie Johnson, \\ PhD, Eli N. Perencevich, MD, MS, Michelle Shardell, PhD, Surbhi Leekha, MD, MPH, and \\ Anthony D. Harris, MD, MPH \\ Departments of Epidemiology and Public Health (DJM, KAT, MS, SL, ADH) and Pathology (JKJ), \\ University of Maryland School of Medicine, Baltimore, MD; the VA Maryland Health Care System \\ (DJM, KAT, ADH), Baltimore, MD; the Department of Epidemiology (ER), University of North \\ Carolina Gillings School of Global Public Health, Chapel Hill, NC; and the University of lowa, \\ Carver College of Medicine \& lowa City VA (ENP), lowa City, IA
}

\section{Abstract}

Objective-To assess the role of environmental contamination in the transmission of multidrugresistant bacteria to healthcare workers' clothing.

Design-Prospective cohort.

Setting-Six intensive care units at a tertiary care hospital.

Subjects-Healthcare workers including registered nurses, patient care technicians, respiratory therapists, occupational/physical therapists, and physicians.

Interventions-None.

Measurements and Main Results-One hundred twenty of 585 (20.5\%) healthcare worker/ patient interactions resulted in contamination of healthcare workers' gloves or gowns. Multidrugresistant Acinetobacter baumannii contamination occurred most frequently, 55 of 167 observations (32.9\%; 95\% confidence interval [CI] $25.8 \%$ to $40.0 \%$ ), followed by multidrug-resistant Pseudomonas aeruginosa, 15 of 86 (17.4\%; 95\% CI 9.4\% to 25.4\%), vancomycin-resistant Enterococcus, 25 of $180(13.9 \%, 95 \%$ CI 8.9, 18.9\%) and methicillin-resistant Staphylococcus aureus, 21 of 152 (13.8\%; 95\% CI 8.3\% to 19.2\%). Independent risk factors associated with healthcare worker contamination with multidrug-resistant bacteria were positive environmental cultures (odds ratio [OR] 4.2; 95\% CI 2.7-6.5), duration in room for $>5$ mins (OR 2.0; 95\% CI 1.2-3.4), performing physical examinations (OR 1.7; 95\% CI 1.1-2.8), and contact with the ventilator (OR 1.8; 95\% CI, 1.1-2.8). Pulsed field gel electrophoresis determined that $91 \%$ of healthcare worker isolates were related to an environmental or patient isolate.

Conclusions-The contamination of healthcare workers' protective clothing during routine care of patients with multidrug- resistant organisms is most frequent with $A$. baumannii. Environmental contamination was the major determinant of transmission to healthcare workers' gloves or gowns. Compliance with contact precautions and more aggressive environmental cleaning may decrease transmission. 


\section{Keywords}

Acinetobacter; contact precautions; contamination; environment; MRSA; VRE

Hospital-associated infections are estimated to contribute to the death of approximately 100,000 people per year in the United States (1). Multidrug-resistant (MDR) bacteria cause a significant proportion of hospital-associated infections (2-4). MDR bacteria are a significant problem worldwide with a high frequency of MDR bacteria in intensive care units (ICUs) from South America, Africa, Asia, and Europe (5-7). MDR Acinetobacter baumannii has emerged as epidemic in many countries (8). MDR bacteria are generally transmitted from patient-to-patient in the healthcare system by transiently contaminated healthcare workers, equipment, and the environment (9).

In multiple smaller studies, looking at one or two organisms, different activities have been associated with a greater likelihood of healthcare worker $(\mathrm{HCW})$ clothing contamination including contact with wound dressing, artificial airways, side rails, linens, infusion pumps, catheters or drain, and direct patient contact including performing a physical examination or spending a longer duration in a room $(4,10,11)$. Studies have not assessed common risk factors for contamination with the most common MDR bacteria and have been limited by clustering of patients or repeated measurements of the same HCW. A. baumannii may be more likely than other MDR bacteria to contaminate HCW clothing or the environment, although it has not been directly compared to methicillin-resistant Staphylococcus aureus (MRSA) or vancomycin-resistant Enterococci (4). Understanding factors that lead to contamination of HCW clothing, and thus increase potential for transmission, may help lead to interventions to prevent transmission of MDR bacteria. To our knowledge, no study has assessed the importance of environmental contamination leading to contamination of $\mathrm{HCW}$ clothing and thus the potential causal role of the environment inpatient-to-patient transmission of MDR bacteria.

To evaluate the differential rate of contamination by MDR A. baumannii compared with other MDR bacteria as well as investigating the importance of environmental contamination in the transfer of MDR bacteria to HCW clothing, we studied a cohort of ICU-based HCWs performing routine patient care.

\section{METHODS}

A cohort study was conducted at the 662-bed University of Maryland Medical Center (Baltimore, MD) between May 26, 2009, and July 31, 2009. All observations occurred in the medical ICU (29-bed unit), surgical ICU (a 19-bed unit), cardiac surgery ICU (a 23-bed unit) or three ICUs in the Shock Trauma Center (totaling 54 trauma ICU beds). The study was approved by the University of Maryland, School of Medicine institutional review board.

We cultured HCWs during routine clinical care activities with patients that were on contact precautions for MDR bacteria. Patients were identified as MDR bacteria colonized or cocolonized on the basis of active surveillance or clinical cultures obtained as part of routine care; MDR bacteria status led to these patients being on contact precautions. Patients selected for study were part of a convenience sample. Patients had to have been identified as having MDR bacteria within the prior year. Active surveillance is performed at the time of admission for MRSA in all units with nares culture, for VRE in the medical ICU and surgical ICU with perirectal cultures, and in all units for MDR A. baumannii when a patient is admitted from another facility (consisting of culturing a patient's sputum, groin, perirectal area, and wound, if present). We do not perform active surveillance for MDR Pseudomonas 
aeruginosa. In our hospital, contact precautions are used for all patients with MRSA and VRE as well as MDR A. baumannii susceptible to two or less antimicrobial classes and MDR $P$. aeruginosa one or less antimicrobial class not including tigecycline or polymyxins. (In this study, all isolates of either species were imipenem-resistant.)

Sequential HCWs (registered nurses, patient care technicians, respiratory therapists, occupational/physical therapists, and physicians) were approached for participation in the study before engaging in routine, nonemergent clinical care activities for these patients. HCW cultures were obtained as described previously $(4,11)$. Briefly, sterile rayon-tipped applicators were moistened with Amies transport media (Becton Dickinson 220093 CultureSwab, liquid Amies, Franklin Lakes, NJ). Hand and glove samples were obtained with a standardized process by swabbing the dorsum of each finger three times and the palm of each hand two times with a twirling motion of the swab with a single swab for both hands or both gloves. Gowns were sampled by swabbing each forearm twice and then swabbing a "W" along the beltline, all with a single swab and performed with a twirling motion. Hands were first sampled before donning gowns and gloves. Data from observations in which $\mathrm{HCW}$ hands were colonized before room entry with the same organism colonizing the patient were excluded from the analysis.

For risk factor collection, before the start of the study, we formed a "work group" of investigators to identify domains of interest for risk factor collection. Risk factors were grouped into domains related to HCW activities, HCW types, type of unit, and patientspecific factors (that could be noted by observation alone). HCW activities were observed and documented including the duration and nature of HCW-patient contact. On completion of activities and before exiting the patient room, HCW gloves and gown were swabbed by investigators. The bare hands of the HCWs were immediately sampled a second time before hand hygiene $(4,11)$.

After enrollment of five HCWs on the same day, using a standardized protocol, environmental sampling was performed on nine sites in the patient room: sink, bed rails, bedside table, vital sign monitor, supply cart, door handle, intravenous pump, ventilator, and floor. At each site, an area of approximately $10 \mathrm{~cm}^{2}$ was sampled using a sterile cotton swab previously moistened with phosphate-buffered saline (2).

For MDR Gram-negative bacteria, swabs were vortexed in $5 \mathrm{~mL}$ of brain heart infusion broth with $6 \mu \mathrm{g} / \mathrm{mL}$ of imipenem (imipenem was added to enhance detection of MDR $A$. baumannii and MDR P. aeruginosa, because all isolates were carbapenem-resistant). For patients known to be colonized with MRSA and VRE, the swabs were placed in brain heart infusion with $2.5 \% \mathrm{NaCl}$. Broths were placed at $35^{\circ} \mathrm{C}$ overnight. For MDR Gram-negatives, $50 \mu \mathrm{L}$ was plated to MacConkey agar with/without $6 \mu \mathrm{g} / \mathrm{mL}$ of imipenem. For MRSA, 50 $\mu \mathrm{L}$ was plated to ChromAgar MRSA and for VRE, $50 \mu \mathrm{L}$ was plated to Enterococcus agar. Plates were incubated $24-48 \mathrm{hrs}$ at $35^{\circ} \mathrm{C}$. Nonlactose fermenting colonies were subcultured to trypticase soy agar with 5\% sheep's blood. Oxidase negative isolates were identified as $A$. baumannii by analytical profile index 20NE test strip or Vitek II (Biomerieux, Durham, NC). Oxidase-positive isolates were subcultured onto Triple Sugar Iron and Pseudomonas (P-agar) slants. Triple Sugar Iron slants were incubated overnight at $35^{\circ} \mathrm{C}$; P-agar slants were placed at $42^{\circ} \mathrm{C}$ overnight. $P$. aeruginosa isolates were identified as $\mathrm{K} / \mathrm{K}$ on Triple Sugar Iron slants and were pyocyanin-producing on P-agar. Colonies of MRSA and VRE were identified using standard laboratory procedures. Susceptibilities were performed following Clinical Laboratory and Standards Institute guidelines.

Pulsed field gel electrophoresis was performed on isolates following protocols from the Centers for Disease Control and Prevention's pulsenet with modifications. Briefly, DNA 
was digested with restriction enzymes including SmaI, ApaI, and SpeI as appropriate according to manufacturer recommendations (New England Biolabs, Beverly, MA [1]). DNA was separated in $1 \%$ agarose on a contour-clamped homogeneous-field machine (CHEF-DR II; Bio-Rad, Richmond, CA). Electrophoresis was performed. After electrophoresis, gels were stained with ethidium bromide and photographed under ultraviolet illumination. Photographic images of gels were saved digitally with the Geldoc EQ (BioRad Laboratories, Hercules, CA) and saved as .tiff files for gel analysis with Gel Compare (Applied Math, Kortrijk, Belgium). The resulting band patterns were compared with the Dice coefficient 100 using the unweighted pair group method to define pulsed field type clusters using the criteria established by Tenover et al (12). Isolates selected for pulsed field gel electrophoresis were those in which patient clinical isolates from the same organism were available to compare with the environmental isolate and $\mathrm{HCW}$ isolate after patient contact. Identical isolates had no band differences, closely related isolates had three or less band difference, partially related had six or less band difference, and unrelated had less than seven band difference as per Tenover et al (12).

Risk factor analysis was conducted using generalized estimating equations to measure the significance of associations between binary variables and the dependent variable of contamination of HCW gowns or gloves with an organism known to be colonizing the patient. Generalized estimating equation allows for control for clustering that would occur because patients or HCWs were repeatedly sampled. Time in the room was nonnormally distributed (mean, 7.9 mins; median, 5.0 mins; interquartile ratio, 3.0-9.0); therefore, time was dichotomized about the median, 5 mins for further analysis to have a more meaningful metric than would be obtained with log transformation. Generalized estimating equations were also used to estimate odds ratios (ORs) and 95\% confidence intervals (CIs) for factors associated with transfer of MDR bacteria to HCW clothing, accounting for nonindependence of patients and adjusting for other risk factors. Potential risk factors were added one by one in the order of most significant $p$ value on bivariable analysis. All statistical tests were twosided; $p<.05$ was considered statistically significant. All analyses were performed using Statistical Analysis Software (SAS), version 9.2 (SAS Corporation, Cary, NC).

\section{RESULTS}

Over $95 \%$ of HCWs approached agreed to participate in the study. We observed 603 unique $\mathrm{HCW}-$ patient interactions. Initial HCW hand samples before patient contact were positive for the same MDR bacteria that the patient was colonized with 3.0\% (18 of 603) of the time; these HCW-patient interactions were subsequently excluded from the final analysis. Of the remaining 585 observed HCW-patient interactions, 180 were for VRE, 167 for MDR $A$.

baumannii, 152 for MRSA, and 86 for MDR $P$. aeruginosa. All HCWs were compliant with contact precautions and hand hygiene after gown and glove removal while under direct observation. No HCWs changed gowns or gloves while in a patient room during this study. Interactions occurred between 36 unique patients over 49 observation periods and 130 unique HCWs for a total of 318 interactions with unique HCWs. Of the 36 patients, 34 had charts available for review. Median age was 61 years (range, 19-90 years), 47\% were female, median length of stay was 33 days (range, 5-260 days), median Charlson comorbidity score was 1 (range, $0-8$ ), and $35 \%$ died in the hospital.

Overall, 120 of 585 (20.5\%) HCW-patient interactions resulted in contamination of HCWs' gloves or gowns. The frequency of detection of VRE, MDR A. baumannii, MRSA, and MDR $P$. aeruginosa on HCW gloves, gowns, or hands after patient care are reported in Table 1. Before room entry, 8.2\% (20 of 245) of HCW room entries had at least one MDR bacteria on their hands. The frequency of specific organisms is presented in Table 1. 
Forty-nine observation periods occurred with individual environmental sampling after each period of observation. Of all rooms sampled, 35 of 49 (71\%) had positive environmental cultures for an MDR organism known to be colonizing the patient. A total of $77.8 \%$ (28 of 36) of rooms housing patients with MDR A. baumannii had environmental cultures for MDR A. baumannii. A total of 35.1\% (13 of 37) of rooms housing patients with VRE had environmental cultures for VRE. A total of $28.1 \%$ (nine of 32) of rooms housing patients with MRSA had environmental cultures for MRSA. A total of $22.2 \%$ (four of 18 ) of rooms housing patients with MDR $P$. aeruginosa had environmental cultures for MDR $P$. aeruginosa.

We were unable to determine how many individual HCWs were contaminated with MDR bacteria, because we did not collect HCW names (130 HCWs indicated it was their first time participating in the study - accounting for 318 interactions with individual HCWs). Pulsed field gel electrophoresis was performed on environmental isolates for which an additional clinical isolate was available from a patient. (Table 2). Clinical isolates for MRSA, MDR $P$. aeruginosa, and MDR $A$. baumannii are routinely saved by the clinical laboratory. There were 22 cases (four MRSA, one MDR P. aeruginosa, and 17 MDR A. baumannii) selected for pulsed field gel electrophoresis, which compared 1) patient clinical isolate; 2) environmental isolate; and 3) HCW isolate after patient contact. Of the 22 cases, 18 (82\%) of HCWs had a strain related to the patient and $20 \mathrm{HCWs}$ had a strain related to the environment.

Risk factors for MDR A. baumannii contamination of HCW gown or gloves after patient care were evaluated with bivariable analysis and then multivariable modeling adjusting for multiple measurements with generalized estimating equations (Table 3). The final model showed that positive environmental culture OR 4.2; 95\% CI 2.7-6.5), duration in room for 5 mins (OR 2.0; 95\% CI 1.2-3.4), performing a physical examination (OR 1.7; 95\% CI 1.12.8 ), and contact with the ventilator (OR 1.8; 95\% CI, 1.1-2.8) were associated with contamination of HCW clothing by MDR bacteria (Table 4).

A model was constructed excluding MDR A. baumannii observations to examine effect of the environment on $\mathrm{HCW}$ contamination with other MDR bacteria. The association of environmental contamination with transmission to HCW clothing was similar (OR 4.3; $p<$. $01)$.

\section{Comment}

Our study found that during routine clinical care of patients with MDR bacteria, HCWs frequently contaminate protective gowns and gloves. This happens with MDR A. baumannii more often than other common MDR bacteria. Approximately one of every three times a $\mathrm{HCW}$ enters the room of a patient with MDR A. baumannii, they will contaminate their gowns or gloves. Some HCWs in ICUs with MDR bacteria have contaminated hands at the time of room entry. Patient rooms were often contaminated with MDR bacteria, especially MDR A. baumannii. We found that environmental contamination was the best predictor of MDR bacteria transmission to $\mathrm{HCW}$ attire.

HCWs frequently become contaminated with MDR bacteria. Using a highly sensitive method, Hayden and colleagues (13) found that $62 \%$ of HCWs who entered the room of patients known to be colonized with VRE contaminated their gloves or hands during care. In experiments of transmission in which investigators touched prespecified areas on a patient or patient room and then imprinted their gloved hands to culture medium, MRSA was found to be transferred to gloves after approximately $30 \%$ to $60 \%$ of contacts $(13,14)$. Using the same method used in the current study with fewer patients and statistical analysis that did not account for clustering, we have examined frequency of contamination with MRSA and 
VRE (11) and MDR $A$. baumannii or MDR $P$. aeruginosa (4). We found a similar frequency of transmission of MDR bacteria in these studies. The impact of contamination of HCW clothing is not precisely understood. Presumably, HCW contamination with MDR bacteria is a key step in transmission to other patients. A study by Duckro et al (15) demonstrated that contaminated HCW hands were capable of transmitting VRE to inanimate surfaces. The frequency that $\mathrm{HCW}$ contamination results in transmission to patients is unknown. In our study, even after appropriate use of gloves and gown while caring for a patient on contact precautions, $1.7 \%$ to $4.2 \%$ of HCWs had a MDR bacterium on their hands without hand hygiene. This emphasizes the importance of hand hygiene after removal of gloves and gown (9).

Overall, $8 \%$ of HCW room entries occurred with a HCW who had MDR bacteria on their hands. The source of contamination of HCW hands may have been prior patient contact or contact with the environment. HCW hand contamination represents a significant potential for transmission and supports Centers for Disease Control and Prevention recommendations to perform hand hygiene before room entry (9). Alternatively, universal use of gown and gloves for all patient contact in the ICU may best address this problem (16).

MDR A. baumannii contaminated HCWs and patient rooms more frequently than other MDR bacteria. MDR A. baumannii was present in $78 \%$ of rooms housing a patient colonized with MDR $A$. baumannii. Similarly, HCWs entering the room of patients had MDR A. baumannii on their hands approximately $4 \%$ of the time, twice as often as other bacteria.

The current study supports the hypothesis that MDR A. baumannii is transmitted more frequently than other MDR bacteria. This increased propensity to contaminate hands, clothing, and the environment may, in part, explain the recent emergence of MDR $A$. baumannii (8). Future research is needed to explore characteristics of MDR A. baumannii that lead to this result.

A greater risk of contamination was observed when HCWs performed a physical examination or remained in the room longer, which are similar to risks of contamination described previously $(4,10,11)$. Contact with a ventilator was associated with a greater risk of contamination, most likely because the respiratory tract is often heavily colonized with MDR bacteria and contact with respiratory equipment may pose a particular risk $(4,10,11)$. The strongest predictor of HCW contamination was a patient room being found to be contaminated with MDR bacteria. HCWs entering a room that had environmental contamination with MDR bacteria were over four times as likely to become contaminated with that organism. Although studies have found an association between environmental cleaning and decreased risk of hospital-associated infections $(17,18)$, the mechanism of and interventions for environmental contamination and transmission of MDR bacteria are not well defined. Our study supports the idea that contamination of a patient's room increases the likelihood of transmission (19-22).

The reason environmental contamination poses such a significant risk for HCW contamination is unclear. In our study, model building included a variable describing number of times a HCW contacted the environment. Frequency of HCW contact with the environment was not significant in the final model, whereas environmental contamination was. This dichotomy is perplexing but an important finding. It may be that patients who contaminate the environment with MDR bacteria are also more likely to contaminate $\mathrm{HCW}$ gowns and gloves through direct patient contact. We did not culture patients for MDR bacteria and cannot address the possibility that patients who contaminated the environment had a greater burden of organism. This should be addressed in future studies. Alternatively, 
contamination of the environment may be the critical step in contamination of HCWs. The latter option would indicate that transmission of MDR bacteria could be significantly decreased with more aggressive daily environmental cleaning. We also did not collect data relative to most recent environmental cleaning, although this would have been done daily, typically within 2-4 hrs of data collection. At a minimum, contamination of the environment appears to be a marker for a patient room at greater likelihood of HCW contamination.

The best method to control the spread of MDR bacteria in the ICU is unknown. Recent studies have focused on detecting and isolating patients for a specific organism such as MRSA (23, 24). Detecting and isolating patients has been studied alone (23) or combined with other interventions, including better hand hygiene, compliance with isolation, and preventive methods for specific healthcare-associated infections (24). Large multicenter studies such as these are necessary to evaluate infection prevention initiatives. Evidence from ours and other studies (13) suggest that environmental cleaning may be appropriate to study in a multicenter intervention trial.

Limitations of this study include that it was a single-center study with a modest number of patient rooms sampled. Because HCW participation was anonymous, we could not adjust for repeated measurements from the same $\mathrm{HCW}$, although we adjusted for multiple observations on the same patient using generalized estimating equations. Although we knew 318 interactions occurred with a HCW on their first experience with the study, we did not know how many times other HCWs may have been observed. Although active surveillance for MDR bacteria is different in different ICUs, this potential bias is unlikely to have had an effect on reported rates of contamination because frequency of transmission did not differ by individual ICU. Although we had a significant detection rate of MDR A. baumannii, our sampling technique may have been insensitive compared with others (25). We did not record time of most recent environmental cleaning and did not assess effectiveness of cleaning. We were unable to assess which or how many patients' anatomic sites were originally positive as a measure of organism burden, which has been associated with transmission of MRSA (10).

In conclusion, MDR A. baumannii was transmitted in approximately one in three interactions with colonized patients, which was much more frequent than other MDR bacteria. Environmental contamination and contamination of HCW hands before entry were twice as common with MDR $A$. baumannii compared with other MDR bacteria, implying that the emergence of MDR A. baumannii may be related to its ability to proliferate and spread on inanimate objects. Transmission of MDR bacteria to HCW clothing was common for all MDR bacteria, emphasizing the importance of improving compliance with contact precautions. Environmental contamination with an MDR bacterium was the major predictive factor in $\mathrm{HCW}$ contamination during patient care suggesting that efforts to decontaminate patient rooms during their stay may decrease transmission.

\section{Acknowledgments}

The authors appreciate the assistance of Jingkun Zhu, MS, for data programming, Lisa Pineles, MA, for editorial assistance and Min Zhan, PhD, for statistical consultation.

This work was funded by an unrestricted research grant from Merck Pharmaceuticals. Other funding was through a 1 K08 HS18111-01 AHRQ to Dr. Morgan, 5K24AI079040-02 National Institutes of Health to Dr. Harris and a VA HSR\&D Merit IIR, 05-123 to Dr. Perencevich. Dr. Perencevich has received unrestricted research grants from Merck and Pfizer. 


\section{References}

1. Thom KA, Johnson JK, Lee M, et al. Environmental contamination of multidrug-resistant Acinetobacter surrounding colonized or infected patients. Am J Infect Control. In press.

2. Hidron AI, Edwards JR, Patel J, et al. NHSN annual update: Antimicrobial-resistant pathogens associated with healthcare-associated infections: Annual summary of data reported to the National Healthcare Safety Network at the Centers for Disease Control and Prevention, 2006-2007. Infect Control Hosp Epidemiol. 2008; 29:996-1011. [PubMed: 18947320]

3. Morgan DJ, Lomotan LL, Agnes K, et al. Characteristics of healthcare-associated infections contributing to unexpected in-hospital deaths. Infect Control Hosp Epidemiol. 2010; 31:864-866. [PubMed: 20569114]

4. Morgan DJ, Liang SY, Smith CL, et al. Frequent multidrug-resistant Acinetobacter baumannii contamination of gloves, gowns, and hands of healthcare workers. Infect Control Hosp Epidemiol. 2010; 31:716-721. [PubMed: 20486855]

5. Vincent JL, Rello J, Marshall J, et al. International study of the prevalence and outcomes of infection in intensive care units. JAMA. 2009; 302:2323-2329. [PubMed: 19952319]

6. Gales AC, Jones RN, Sader HS. Contemporary activity of colistin and polymyxin B against a worldwide collection of Gram-negative pathogens: Results from the SENTRY Antimicrobial Surveillance Program(2006-09). J Antimicrob Chemother. 2011; 66:2070-2074. [PubMed: 21715434]

7. Biedenbach DJ, Bell JM, Sader HS, et al. Antimicrobial susceptibility of Gram-positive bacterial isolates from the Asia-Pacific region and an in vitro evaluation of the bactericidal activity of daptomycin, vancomycin, and teicoplanin: A SENTRY Program Report (2003-2004). Int J Antimicrob Agents. 2008; 30:143-149. [PubMed: 17531446]

8. Peleg AY, Seifert H, Paterson DL. Acinetobacter baumannii: Emergence of a successful pathogen. Clin Microbiol Rev. 2008; 21:538-582. [PubMed: 18625687]

9. Siegel JD, Rhinehart E, Jackson M, et al. Health Care Infection Control Practices Advisory Committee:2007 Guideline for isolation precautions: Preventing transmission of infectious agents in health care settings. Am J Infect Control. 2007; 35(Suppl 2):S65-S164. [PubMed: 18068815]

10. Boyce JM, Potter-Bynoe G, Chenevert C, et al. Environmental contamination due to methicillinresistant Staphylococcus aureus: Possible infection control implications. Infect Control Hosp Epidemiol. 1997; 18:622-627. [PubMed: 9309433]

11. Snyder GM, Thom KA, Furuno JP, et al. Detection of methicillin-resistant Staphylococcus aureus and vancomycin-resistant Enterococci on the gowns and gloves of healthcare workers. Infect Control Hosp Epidemiol. 2008; 29:583-589. [PubMed: 18549314]

12. Tenover FC, Arbeit RD, Goering RV, et al. Interpreting chromosomal DNA restriction patterns produced by pulsed-field gel electrophoresis: Criteria for bacterial strain typing. J Clin Microbiol. 1995; 33:2233-2239. [PubMed: 7494007]

13. Hayden MK, Bonten MJ, Blom DW, et al. Reduction in acquisition of vancomycin-resistant Enterococcus after enforcement of routine environmental cleaning measures. Clin Infect Dis. 2006; 42:1552-1560. [PubMed: 16652312]

14. Stiefel U, Cadnum JL, Eckstein BC, et al. Contamination of hands with methicillin-resistant Staphylococcus aureus after contact with environmental surfaces and after contact with the skin of colonized patients. Infect Control Hosp Epidemiol. 2011; 32:185-187. [PubMed: 21460476]

15. Duckro AN, Blom DW, Lyle EA, et al. Transfer of vancomycin-resistant Enterococci via health care worker hands. Arch Intern Med. 2005; 165:302-307. [PubMed: 15710793]

16. Matsushima A, Tasaki O, Tomono K, et al. Pre-emptive contact precautions for intubated patients reduced healthcare-associated methicillin-resistant Staphylococcus aureus transmission and infection in an intensive care unit. J Hosp Infect. 2011; 78:97-101. [PubMed: 21474201]

17. Hota B, Blom DW, Lyle EA, et al. Interventional evaluation of environmental contamination by vancomycin-resistant Enterococci: Failure of personnel, product, or procedure? J Hosp Infect. 2009; 71:123-131. [PubMed: 19108932] 
18. Datta R, Platt R, Yokoe DS, et al. Environmental cleaning intervention and risk of acquiring multidrug-resistant organisms from prior room occupants. Arch Intern Med. 2011; 171:491-494. [PubMed: 21444840]

19. Boyce JM. Environmental contamination makes an important contribution to hospital infection. J Hosp Infect. 2007; 65(Suppl 2):50-54. [PubMed: 17540242]

20. Carling PC, Bartley JM. Evaluating hygienic cleaning in health care settings: What you do not know can harm your patients. Am J Infect Control. 2010; 38(Suppl 1):S41-S50. [PubMed: 20569855]

21. Dancer SJ. The role of environmental cleaning in the control of hospital- acquired infection. J Hosp Infect. 2009; 12 73:378-385. [PubMed: 19726106]

22. Nseir S, Blazejewski C, Lubret R, et al. Risk of acquiring multidrug-resistant Gram-negative bacilli from prior room occupants in the intensive care unit. Clin Microbiol Infect. 2011; 17:12011208. [PubMed: 21054665]

23. Huskins WC, Huckabee CM, O'Grady NP, et al. Intervention to reduce transmission of resistant bacteria in intensive care. N Engl J Med. 2011; 364:1407-1418. [PubMed: 21488763]

24. Jain R, Kralovic SM, Evans ME, et al. Veterans Affairs initiative to prevent methicillin-resistant Staphylococcus aureus infections. N Engl J Med. 2011; 364:1419-1430. [PubMed: 21488764]

25. Hayden MK, Blom DW, Lyle EA, et al. Risk of hand or glove contamination after contact with patients colonized with vancomycin-resistant Enterococcus or the colonized patients' environment. Infect Control Hosp Epidemiol. 2008; 29:149-154. [PubMed: 18179370] 


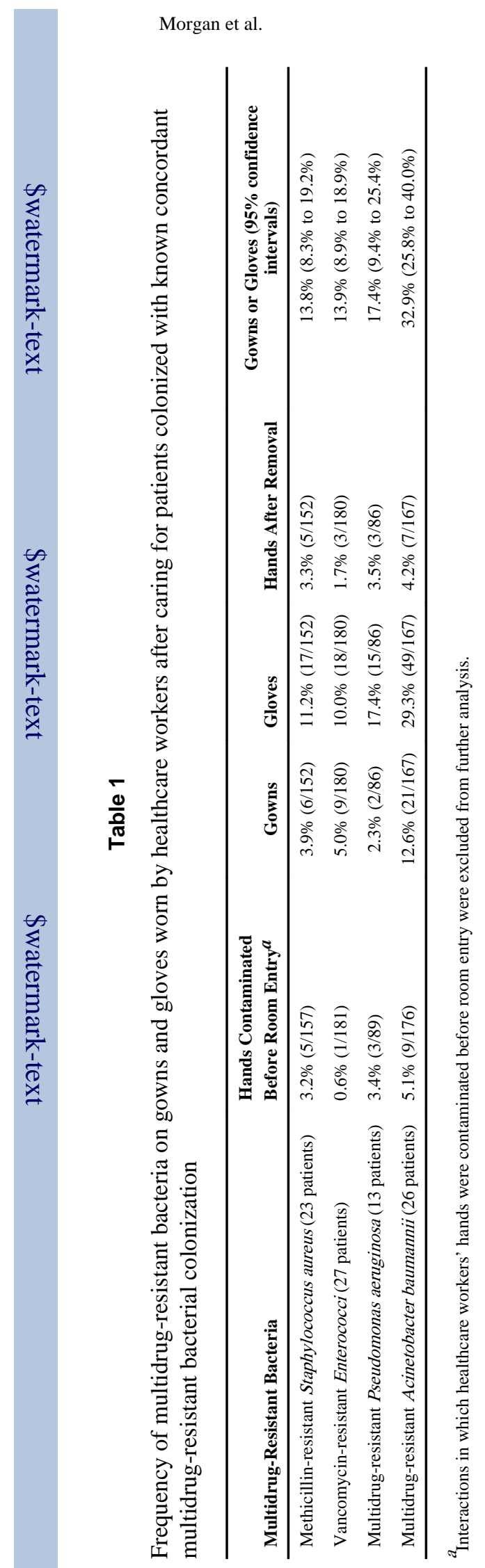

Page 10 
Table 2

Relatedness of multidrug-resistant bacterial isolates from healthcare workers' gowns or gloves, patient clinical isolate, and patient room environmental isolate

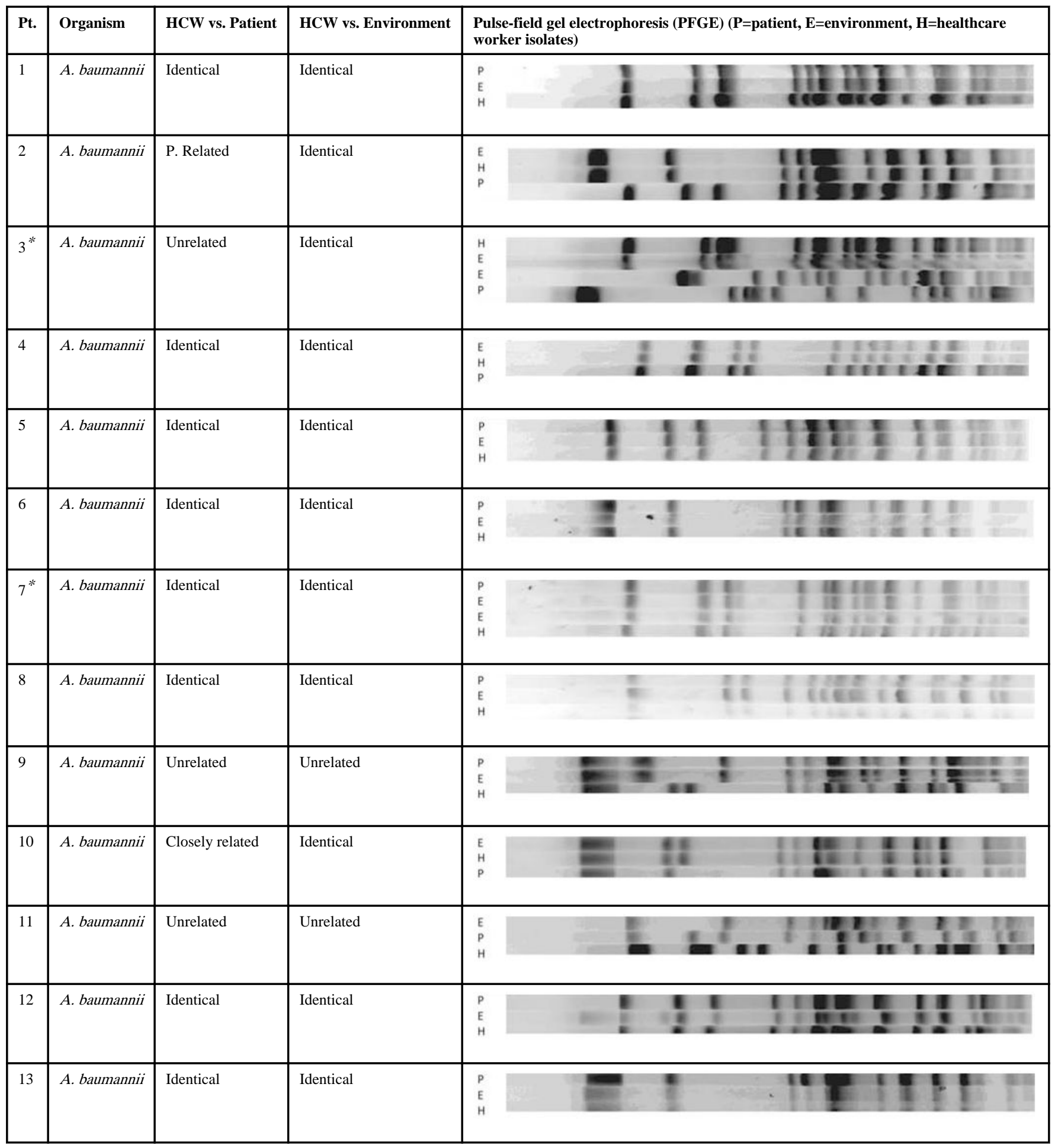




\begin{tabular}{|c|c|c|c|c|}
\hline Pt. & Organism & HCW vs. Patient & HCW vs. Environment & $\begin{array}{l}\text { Pulse-field gel electrophoresis (PFGE) (P=patient, } E=\text { =environment, } \mathrm{H}=\text { healthcare } \\
\text { worker isolates) }\end{array}$ \\
\hline 14 & A. baumannii & Closely related & Identical & $=i \frac{1}{2}$ \\
\hline 15 & A. baumannii & Unrelated & Identical & $\begin{array}{l}\mathrm{H} \\
\mathrm{E} \\
\mathrm{p}\end{array}$ \\
\hline 16 & A. baumannii & Identical & Identical & 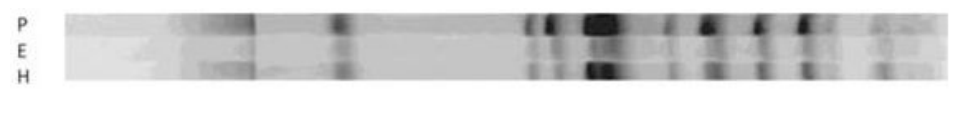 \\
\hline 17 & A. baumannii & Identical & Identical & 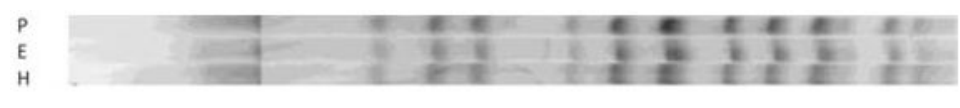 \\
\hline 18 & $P$. aeruginosa & Identical & Identical & ชะ \\
\hline 19 & MRSA & Identical & Closely related & 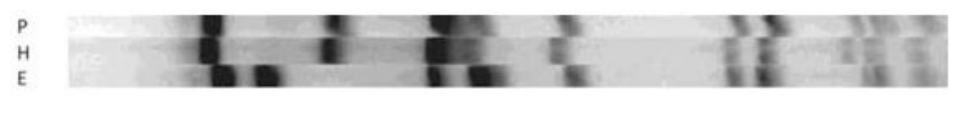 \\
\hline 20 & MRSA & Identical & Identical & 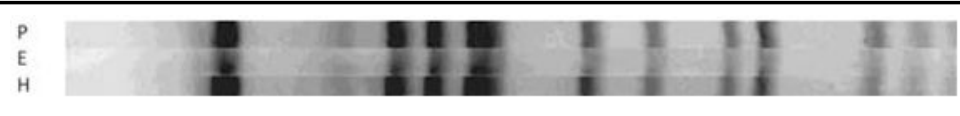 \\
\hline 21 & MRSA & Identical & Identical & 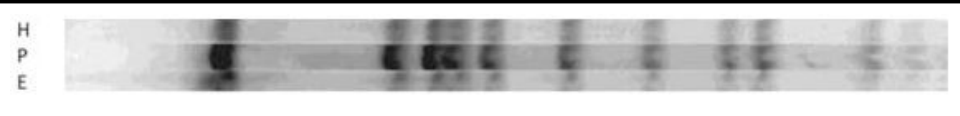 \\
\hline 22 & MRSA & Partially related & Partially related & $\begin{array}{l}\mathrm{P} \\
\mathrm{E}\end{array} \mathrm{A}$ \\
\hline
\end{tabular}

MRSA, methicillin-resistant Staphylococcus aureus; P, patient; E, environment; H, healthcare worker isolates.

Single environmental culture had more than one strain of multidrug-resistant $A$. baumannii; pulsed field gel electrophoresis performed on both isolates. 
Table 3

Proportion of detection of multidrug-resistant multidrug-resistant bacteria on the gowns and gloves worn by healthcare workers caring for patients know to be colonized with concordant multidrug-resistant bacteria (i.e., same type of multidrug-resistant bacterial colonization as detected on gowns and gloves after leaving patient room $)^{a}$

\begin{tabular}{|c|c|c|c|}
\hline \multirow[b]{2}{*}{ Variable } & \multicolumn{3}{|c|}{ Observations } \\
\hline & $\begin{array}{l}\text { Percent With Positive Culture } \\
\text { Results }(n=124)\end{array}$ & $\begin{array}{c}\text { Percent With Negative Culture } \\
\text { Results }(n=461)\end{array}$ & Significance $(p)^{b}$ \\
\hline \multicolumn{4}{|l|}{ Healthcare worker activities in room } \\
\hline Use of paper gown vs. vinyl & $43 \%(53)$ & $47 \%(216)$ & .76 \\
\hline Bathing & $12 \%(1)$ & $1 \%(39)$ & .42 \\
\hline Bedrail & $51 \%(63)$ & $47 \%(217)$ & .24 \\
\hline Bedside table & $21 \%(26)$ & $17 \%(80)$ & .11 \\
\hline Catheter drain & $20 \%(25)$ & $18 \%(84)$ & .89 \\
\hline Door handle & 0 & $1 \%(3)$ & N/A \\
\hline Floor & $8 \%(10)$ & $5 \%(23)$ & .45 \\
\hline Intravenous pump & $31 \%(38)$ & $29 \%(135)$ & .20 \\
\hline Intravenous tubing & $32 \%(40)$ & $31 \%(145)$ & .54 \\
\hline Oral medications & $9 \%(11)$ & $6 \%(27)$ & .19 \\
\hline Physical examination & $71 \%(88)$ & $58 \%(268)$ & .02 \\
\hline Sink & $10 \%(13)$ & $8 \%(36)$ & .25 \\
\hline Supply cart & $51 \%(63)$ & $49 \%(226)$ & .96 \\
\hline Ventilator & $27 \%(33)$ & $17 \%(79)$ & $<.01$ \\
\hline Vital sign monitor & $11 \%(14)$ & $9 \%(40)$ & .79 \\
\hline Vital signs & $31 \%(38)$ & $31 \%(141)$ & .84 \\
\hline Wound dressing & $10 \%(13)$ & $5 \%(24)$ & .03 \\
\hline Contact endotracheal tube/tracheostomy & $33 \%(41)$ & $22 \%(101)$ & .37 \\
\hline Duration in room ( $>5$ mins) & $65 \%(81)$ & $49 \%(224)$ & $<.01$ \\
\hline No. of patient contacts (>3) & $56 \%(70)$ & $45 \%(209)$ & $<.01$ \\
\hline No. of environmental contacts $(>3)$ & $46 \%(57)$ & $40 \%(185)$ & .11 \\
\hline \multicolumn{4}{|l|}{ Patient-specific factors } \\
\hline Endotracheal tube & $35 \%(43)$ & $25 \%(117)$ & $<.01$ \\
\hline Tracheostomy & $65 \%(80)$ & $77 \%(354)$ & .57 \\
\hline Patient urinary catheter & $90 \%(111)$ & $90 \%(417)$ & .68 \\
\hline Patient unit & & & .74 \\
\hline Medical intensive care unit & $63 \%(78)$ & $57 \%(264)$ & \\
\hline Surgical intensive care unit & $15 \%(18)$ & $18 \%(84)$ & \\
\hline Other location & $23 \%(28)$ & $25 \%(113)$ & \\
\hline Healthcare worker type & & & .15 \\
\hline Nurse & $54 \%(67)$ & $50 \%(231)$ & \\
\hline Physician & $12 \%(15)$ & $19 \%(86)$ & \\
\hline Personal care technician & $10 \%(13)$ & $13 \%(62)$ & \\
\hline Respiratory therapist & $17 \%(21)$ & $10 \%(48)$ & \\
\hline
\end{tabular}




\begin{tabular}{|c|c|c|c|}
\hline \multirow[b]{2}{*}{ Variable } & \multicolumn{3}{|c|}{ Observations } \\
\hline & $\begin{array}{l}\text { Percent With Positive Culture } \\
\text { Results }(n=124)\end{array}$ & $\begin{array}{c}\text { Percent With Negative Culture } \\
\text { Results }(n=461)\end{array}$ & Significance $(p)^{b}$ \\
\hline Other provider & $6 \%(8)$ & $7 \%(34)$ & \\
\hline Environment-positive concordant organism & $70 \%(87)$ & $34 \%(156)$ & $<.01$ \\
\hline
\end{tabular}

N/A, not applicable.

$a_{\text {Italicized variables are those considered in the final regression model; }}$

$b_{\text {from generalized estimating equations. }}$ 


\section{Table 4}

Variables found to be independently predictive of healthcare worker contamination with multidrug-resistant bacteria

\begin{tabular}{lrr}
\hline Independent Variable & Odds Ratio (95\% Confidence Interval) & $\boldsymbol{p}^{\boldsymbol{a}}$ \\
\hline Positive multidrug-resistant bacteria environmental culture & $4.15(2.66-6.47)$ & $<.001$ \\
Duration in room $>$ 5 mins & $1.99(1.15-3.43)$ & .014 \\
Performing physical examination & $1.74(1.10-2.77)$ & .019 \\
Contact with ventilator & $1.78(1.12-2.82)$ & .014 \\
\hline
\end{tabular}

${ }^{a}$ From generalized estimating equations. 